\title{
Hybrid approaches to nanometer-scale patterning: Exploiting tailored intermolecular interactions
}

\author{
Thomas J. Mullen · Charan Srinivasan • \\ Mitchell J. Shuster · Mark W. Horn • \\ Anne M. Andrews · Paul S. Weiss
}

Received: 20 December 2007 / Accepted: 27 March 2008/Published online: 23 May 2008

(C) Springer Science+Business Media B.V. 2008

\begin{abstract}
In this perspective, we explore hybrid approaches to nanometer-scale patterning, where the precision of molecular self-assembly is combined with the sophistication and fidelity of lithography. Two areas-improving existing lithographic techniques through self-assembly and fabricating chemically patterned surfaces-will be discussed in terms of their advantages, limitations, applications, and future outlook. The creation of such chemical patterns enables new capabilities, including the assembly of biospecific surfaces to be recognized by, and to capture analytes from, complex mixtures. Finally, we speculate on the potential impact and upcoming challenges of these hybrid strategies.
\end{abstract}

T. J. Mullen · M. J. Shuster · P. S. Weiss $(\bowtie)$ Departments of Chemistry and Physics, The Pennsylvania State University, University Park, PA 16802-6300, USA e-mail: stm@psu.edu

C. Srinivasan · M. W. Horn

Department of Engineering Science and Mechanics, The Pennsylvania State University, University Park, PA 16802-6300, USA

A. M. Andrews ( $\square)$

Department of Veterinary and Biomedical Science and Huck Institutes of the Life Sciences, The Pennsylvania State University, University Park, PA 16802-6300, USA e-mail: ama11@psu.edu
Keywords Self-assembly · Nanolithography · Chemical patterning - Soft lithography · Intermolecular Interactions · Future science challenges

\section{Introduction}

Currently, one of the great engineering challenges is to gain the ability to fabricate nanoscale structures at the supramolecular (1-50 nm) length scale with high precision, throughput, and reproducibility. In microelectronics, speed and density have been the driving forces to increase the final resolution of traditional topdown methodologies utilizing deposition, etching, or modification of thin layers on semiconductor substrates. However, the impetus to create features with molecularscale structures, properties, and interactions has motivated and expanded research into fields outside traditional semiconductor nanofabrication. One approach has been the development of hybrid patterning strategies for a wide range of applications. These utilize self- and directed assembly in conjunction with existing nanofabrication infrastructure (Xia et al. 1999; Lewis et al. 2001a; Smith et al. 2004; Srinivasan et al. 2007).

The initial motivation for the development of microand nanoscale patterning came from the fabrication needs of the semiconductor industry. In 1965, when Moore first predicted that the number of components on a silicon wafer would double every year and a half, there were only 30 microscale transistors on a single 
microprocessor, and the extrapolated growth was expected for a single decade (Moore 1965). Amazingly, this trend, referred to as "Moore's Law," has been sustained for over 40 years and silicon substrates now contain billions of nanoscale devices (Moore 1995). To attain nanoscale features, the complexity and the cost of nanofabrication facilities have grown tremendously. Figure 1 shows the growth of the cost of a semiconductor fabrication facility over the past 30 years (Thompson and Parthasarathy 2006). If this trend were to continue for the next half century, the cost of a single facility would be greater than the current gross domestic product of the United States (Mannering and Hodge 2007). Over the same time period, the price-per-transistor has decreased seven-fold, enabling cheaper, smaller, and more efficient devices. These two factors underlie many of the advances in the semiconductor industry (Thompson and Parthasarathy 2006). However, this type of scaling will eventually taper off, not only because of fabrication costs, but also due to the physical limitations of the materials and methods currently used to create semiconductor nanoscale features (Lundstrom 2003). In contrast, selfassembly methodologies exploit the inherent chemical and physical properties of molecules to direct and to control their arrangements and locations on surfaces with nanometer or better precision. By engineering molecules with varying structures, intermolecular interaction strengths, and terminal groups, the properties of these chemical films, such as surface reactivity and screening properties, can be tailored (Kumar et al. 1994; Smith et al. 2001; Smith et al. 2004; Dameron et al. 2005a). Despite the ability of self-assembly strategies to control the structures of chemical films, the direct placement and the fabrication of complex multicomponent structures via bottom-up assembly are limited and require further development to become viable alternatives to traditional lithographic techniques (Xia et al. 1999; Mullen et al. 2007b).

In this perspective, we specifically examine and highlight the advantages, limitations, applications, and future outlook of several hybrid patterning technologies where the sophistication and control of lithography are coupled with the molecular precision of self-assembly. A number of comprehensive reviews on hybrid patterning strategies have been published (Xia et al. 1999; Hammond 2004; Gates et al. 2005; Love et al. 2005; Rogers and Nuzzo 2005; Henzie et al. 2006; Saavedra et al. 2008). We first describe

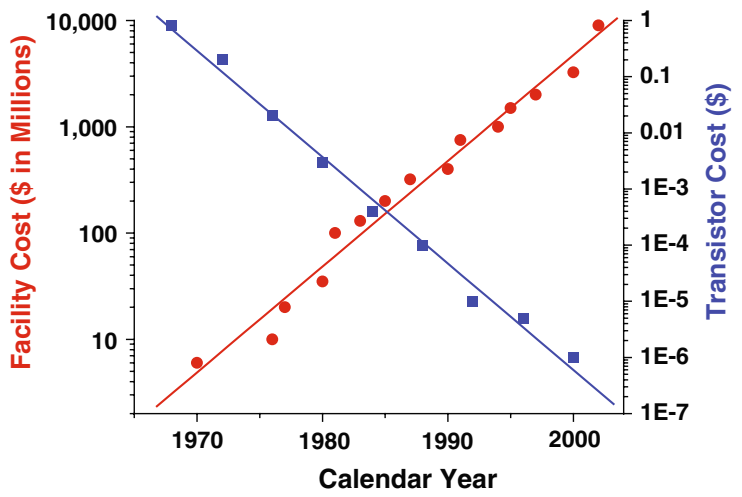

Fig. 1 Traditional semiconductor costs. The cost of a single semiconductor fabrication facility (red circles) and a single transistor (blue squares) over the past 30 years is shown. Graph is adapted from Thompson and Parthasarathy 2006

how self-assembly can be used to improve the resolution of existing lithographic technologies. Next, we discuss chemically patterned surfaces created by hybrid strategies where the strengths are maximized and the limitations are minimized in terms of ease of use, reproducibility, resolution, and precision. Finally, we explore the potential impact of and future outlook for hybrid strategies.

\section{Structures with molecular precision}

Initially, we anticipate that hybrid strategies will need to be adapted to transfer the intrinsic molecular precision of self-assembly to established nanofabrication infrastructure and technology. Although the placement and alignment of microscale structures with nanometer-scale precision is difficult using most lithographic techniques, the additional requirement of patterning over large areas (tens of $\mathrm{cm}^{2}$ ) for industrial applications makes it extremely challenging using only current top-down strategies (Henzie et al. 2006). For example, photolithography is commonly used to fabricate surface features for integrated circuits in semiconductor manufacturing because of its ability to create reproducible structures with high throughput and relatively low cost (Rai-Choudhury 1997). This parallel methodology fabricates surface structures by patterning a light-sensitive polymer via photon exposure through a mask. The pattern is then developed via wet chemistry methods and etched into the underlying substrate. However, feature sizes resulting from 
photolithography are diffraction limited at $\sim 100 \mathrm{~nm}$ without resolution enhancement techniques (Brunner 2003). To fabricate smaller features, electron-beam lithography has been utilized. This technology employs high-energy electrons to write a pattern directly into an electron-sensitive polymer, which is then developed, translating the pattern into the underlying substrate. Electron-beam lithography has higher resolution than photolithography, and features down to $\sim 20 \mathrm{~nm}$ can now be routinely fabricated. However, because electron-beam lithography is a serial technique, it is expensive and slow compared to photolithography, and thus, is not practical for industrial-scale fabrication applications except in specific highly leveraged circumstances such as photolithographic mask fabrication (Brunner 2003). With hybrid techniques, molecular-scale features can be rapidly created over large areas using conventional top-down lithography to create microscopic features combined with molecular self-assembly to control supramolecular organization (Smith et al. 2004).

One example of this type of hybrid strategy is the molecular-ruler process, where conventional lithography is coupled with selective deposition of multilayers of bifunctional organic molecules and coordinated metal ions. Ultimately, the chemical multilayer film defines the nanometer-scale spacings of the lithographically patterned surface structures (Evans et al. 1991; Hatzor and Weiss 2001; Haes et al. 2004). Figure 2 shows a schematic of the molecular-ruler assembly process. Initially, a lithographically defined gold parent structure is fabricated on an oxidized $\mathrm{Si}$ substrate. The molecular ruler, consisting of sequential alternating layers of $\alpha, \omega$ mercaptoalkanoic acid and cupric ions $\left(\mathrm{Cu}^{2+}\right)$, is then deposited onto the gold parent structure. After the desired thickness of the molecular-ruler stack is achieved via multiple molecular layer deposition steps, a daughter metal is deposited across the entire substrate. The molecular ruler, along with the daughter metal atop the molecular resist, is removed by chemical lift-off, leaving precisely defined spacings $(4-100 \mathrm{~nm})$ between the parent and daughter structures. This scheme has the advantage that defects in SAMs (Tiberio et al. 1993; Poirier and Pylant 1996; Jager et al. 1997; Bent 2007) are mitigated through multilayer assembly via the varying stoichiometry possible between the molecules and the ionic ligands (Daniel et al. 2007; Hatzor de Picciotto et al. (a)

\section{parent metal}

Si substrate

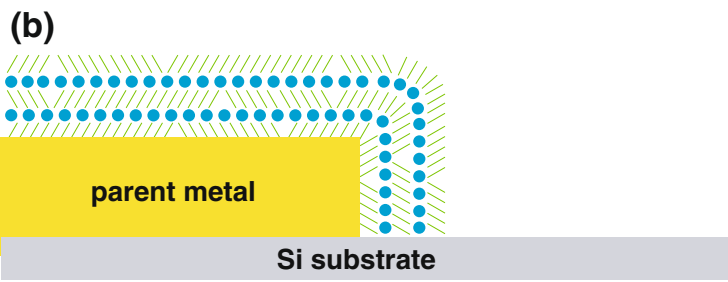

(c)

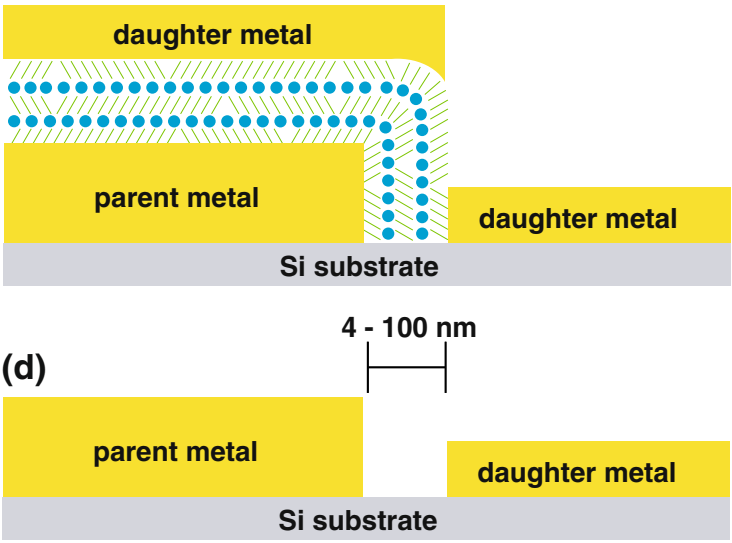

Fig. 2 Molecular-ruler assembly process. (a) Initially, a gold parent structure is fabricated by conventional lithography. (b) Subsequently, the molecular ruler, consisting of alternating layers of $\alpha, \omega$-mercaptoalkanoic acid (green lines) and cupric ions $\left(\mathrm{Cu}^{2+}\right.$, blue circles), is deposited onto the gold parent structure until the desired multilayer thickness is achieved. (c) Daughter metal is then deposited across the entire substrate. (d) The molecular ruler and the daughter metal atop of it are removed with a chemical lift-off process, leaving a precisely defined spacing $(1-100 \mathrm{~nm})$ between the parent and daughter structures. Schematic is not to scale

2007). This process can also be combined with photolithography (Anderson et al. 2006) or electronbeam lithography (Tanaka et al. 2004) to create patterns at multiple scales. This combination of established patterning methods with a novel chemical processing technique demonstrates the compatibility and robustness of hybrid strategies and holds promise for further miniaturizing electronic devices.

As opposed to exploiting chemical multilayer films to create precise supramolecular spacings, self-assembled block copolymers, consisting of covalently bonded hydrophobic and hydrophilic units, can 
enhance the critical dimensions and fidelity of surface features fabricated by lithography (Mansky et al. 1997; Park et al. 1997; Thurn-Albrecht et al. 2000; Hawker and Russell 2005; Stoykovich and Nealey 2006; Black 2007; Stoykovich et al. 2007). Figure 3 depicts the use of block-copolymer-directed assembly to improve the line-edge roughness of a pattern fabricated by conventional lithography. Initially, chemical functionality is patterned on a substrate by conventional lithography, such as electron-beam lithography, and subsequent oxygen plasma treatment. A thin film $(<100 \mathrm{~nm})$ of block copolymer is then cast across the patterned substrate and thermally treated such that microphase separation of the block copolymer produces domains that register with the underlying substrate. However, because the blockcopolymer assembly is thermodynamically controlled, the defects and irregularities in the underlying chemical pattern are self-corrected in the block-copolymer layer. Finally, the enhanced registration of the block copolymer domains is transferred into the underlying substrate (Stoykovich et al. 2005). This self-healing process is in contrast to current chemically amplified resists, which are based on diffusion-limited processes, where the final patterned features are sensitive to small variations in processing conditions (Tanaka et al. 1998).

Self-assembled monolayers (SAMs) can also spontaneously phase separate into nanoscale domains when two or more molecular species with differing intermolecular interaction strengths are coadsorbed (Stranick et al. 1994; Lewis et al. 2001b; Smith et al. 2001). These interaction strengths can be controlled by selecting the head groups, backbones, and/or tail groups of the deposited molecules (Mullen et al. 2007b). Further, nanoscale domains can be artificially fabricated and manipulated by exploiting the molecular exchange into and displacement of a labile monolayer (Bumm et al. 1999; Dameron et al. 2005a; Mullen et al. 2006). This displacement process has been exploited to improve existing chemical patterning strategies (vide infra). Even small multicomponent patches of molecules $(\sim 15 \mathrm{~nm})$ can be made to separate on the nanoscale (Salaita et al. 2005). In many cases, phase separation can be controlled by the selection of the molecules and processing conditions, which determine the dynamics of the structures created (Bumm et al. 1999, Smith et al. 2004). As with block copolymers, separated SAMs enable chemical

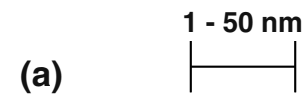

\section{Si substrate}

(b)

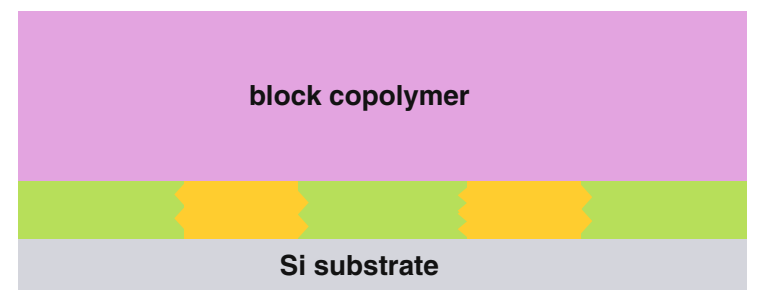

(c)

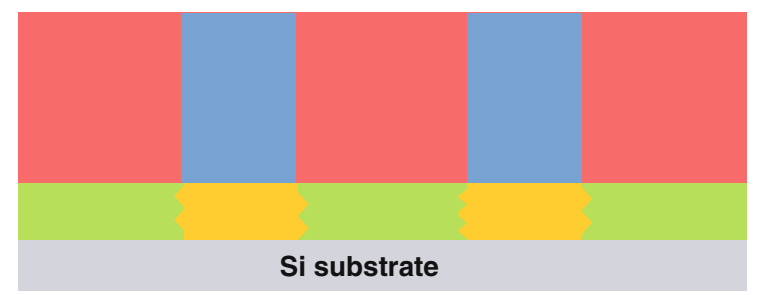

(d)

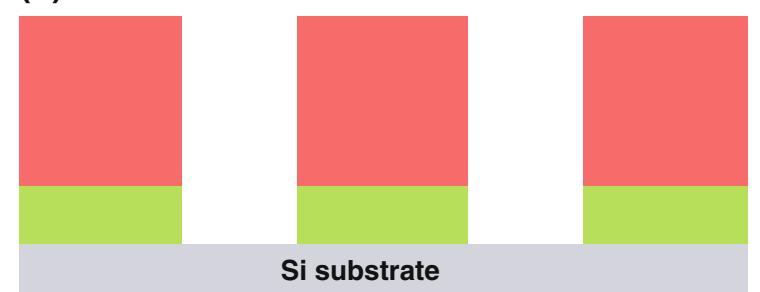

Fig. 3 Block-copolymer-directed assembly. (a) A chemical pattern is placed across a substrate via conventional lithography. (b) Next, a thin film $(<100 \mathrm{~nm})$ of block copolymer is deposited onto the patterned surface and (c) thermally annealed such that microphase separation of the block copolymer produces domains that align with the patterned chemical surface. (d) The registration and alignment of the block-copolymer domains are translated with molecular-scale precision onto the underlying substrate. Schematic is not to scale

patterns to be created at the few to tens of nanometers scale.

These examples highlight how self-assembly might be used to improve and to enhance existing lithography techniques. An advantage of these hybrid techniques is their ability to interface with and to utilize existing infrastructure. Another advantage is that these techniques are independent of both resolution and the top-down patterning methods employed, allowing them to be adapted to future lithographic technologies. 
However, despite the advantages of these hybrid strategies, there are challenges that prevent their immediate translation into industry. First, the majority have only been developed recently and for specialized applications. It is difficult for the semiconductor industry to adopt techniques that have not been demonstrated and optimized for its own specific applications. Additionally, in some instances, the selfand directed assembly strategies employed in hybrid patterning are not yet compatible with the types of materials and processes used in existing semiconductor infrastructure. While efforts have been made to migrate these strategies so that they can be applied directly to technological materials, much remains to be done on this front (Bent 2007).

One of the ultimate goals of combining the intrinsic molecular precision of self-assembly with lithography is to fabricate precise features and to produce structures with higher resolution and lower production costs than structures made using conventional lithographic techniques alone. The molecular-ruler process demonstrates the utility of multilayer chemical films and how they can be used to create precise and proximate nanoscale structures. With self-assembling block copolymers, the domains of the block copolymer enhance the pattern transfer into the underlying substrate. Currently, self-assembled block copolymers are not aimed at improving the resolution of conventional lithography, but rather are intended to improve process control, such as line-edge roughness, and information transfer from the exposure tool to the substrate. In the near term, the continuing research goals for coupling self-assembly to lithography are to develop more diverse approaches to fabricate precise nanoscale structures. By identifying the advantages and limitations of different strategies, the potential for large-scale implementation can be advanced.

\section{Chemically patterned surfaces}

Above, we had described how self-assembly can be used to improve existing lithographic techniques. Hybrid strategies are also being employed to create chemically patterned surfaces that make possible a range of new applications. A recent example is the fabrication of biospecific surfaces that recognize and capture specific biomolecules from complex environments. Functionalization of chemically patterned surfaces adds new degrees of utility by enabling surface reactivity to be patterned at the nanometer scale. This has led to nanoscale films being used as biocompatible/bioactive scaffolds, molecular-sized electronic components, selective molecular resists, and other types of surfaces where both patterned structure and molecular interactions are necessary (Chen et al. 1997; Xia and Whitesides 1998a; Smith et al. 2004; Srinivasan et al. 2007). As processes are developed for creating such chemical patterns, metrology tools and methods must be developed in lockstep in order to follow the patterning steps and to optimize the quality of the surfaces obtained. These metrology methods remain in their infancy (Allara and Nuzzo 1985; Porter et al. 1987; Nuzzo et al. 1990; Lopez et al. 1993; Pertsin and Grunze 1994; Lahiri et al. 1999a; Srinivasan et al. 2007; Mrksich 2008).

One technique to fabricate chemically patterned surfaces is lithography-assisted chemical patterning (LACP), where conventional lithography is employed in conjunction with SAMs to create chemical patterns with high fidelity (Anderson et al. 2006; Srinivasan et al. 2007). This is accomplished by exploiting a commercially available lift-off resist that can withstand the selfassembly process without disrupting the underlying SAM. Figure 4a shows an example of the LACP process. Initially, a bilayer resist consisting of an underlying lift-off resist and an overlayer photoresist is cast over a preexisting SAM. This bilayer resist is then patterned via conventional lithography. The photoresist is then removed, leaving behind the underlying lift-off resist; the lift-off resist withstands the solvents used in further self-assembly. Exposed regions can be stripped of the existing monolayer and backfilled with a new monolayer, or molecules can be inserted into the exposed regions of the SAM via solution deposition. The lift-off resist is then removed, leaving the original SAM undisturbed in the underlying regions. Because of the close integration with conventional lithography, LACP is capable of creating 1:1 registered chemical patterns over large areas and enables feature dimensions to be readily scaled down, which is difficult by other chemical patterning strategies.

Soft lithography is another strategy used to fabricate chemically patterned surfaces. This encompasses microcontact printing $(\mu \mathrm{CP})$ and related techniques. In $\mu \mathrm{CP}$, an elastomeric stamp coated with molecules is applied to a substrate, and a chemical pattern is formed in the regions where the stamp and substrate are in contact (Kumar and Whitesides 1993; Xia and 
(a)

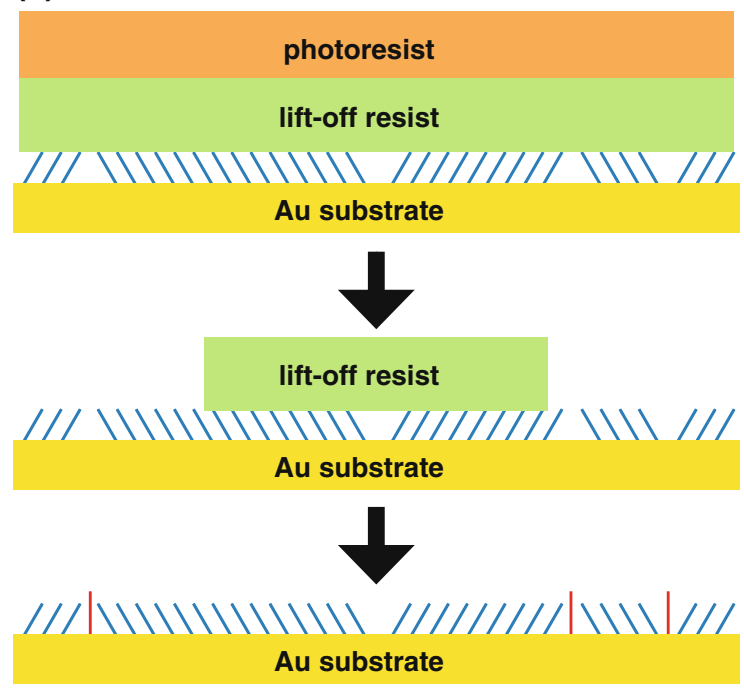

(b)

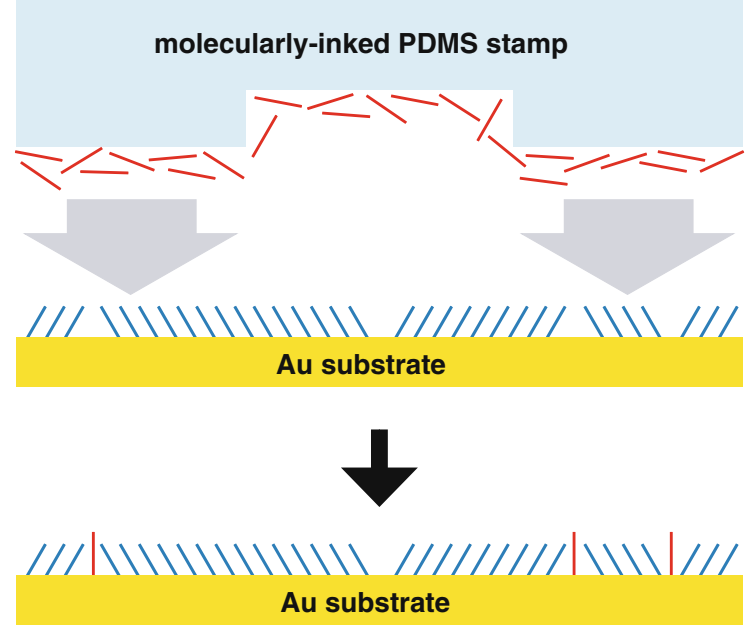

Fig. 4 (a) Lithography-assisted chemical patterning. A bilayer resist consisting of lift-off resist and photoresist is deposited across a preexisting SAM and patterned via conventional lithography, leaving behind a chemically robust lift-off layer. Molecules are then inserted into the SAM via solution deposition in regions not protected by the lift-off resist. Finally, the remaining lift-off resist is removed, leaving a patterned SAM. (b) Microcontact insertion printing. A patterned elastomeric stamp coated with the molecules to be patterned is brought into contact with a substrate coated with a preexisting monolayer that is not easily displaced; the molecules on the stamp insert into the defects in the preexisting monolayer where the stamp and substrate are in contact. Schematics are not to scale

Whitesides 1998a; Xia and Whitesides 1998b; Smith et al. 2004). One limitation of $\mu \mathrm{CP}$ is the requirement for patterned molecules to have sufficient intermolecular interaction strengths to prevent lateral diffusion and hence pattern dissolution, both during and after patterning (Delamarche et al. 1998; Dameron et al. 2005b). Microdisplacement printing ( $\mu \mathrm{DP}$ ) circumvents this limitation by utilizing a preexisting monolayer that is sufficiently labile when exposed to other thiolated molecules via the stamping process but nonetheless prevents lateral diffusion of the patterned molecules (Dameron et al. 2005b, c; Srinivasan et al. 2007). However, in both $\mu \mathrm{CP}$ and $\mu \mathrm{DP}$, the molecular composition of a patterned film is determined by and restricted to the feature size of the elastomeric stamp. In contrast, the molecular composition of chemically patterned films created by microcontact insertion printing $(\mu \mathrm{CIP})$ is controlled by other factors such as the density of defects in the initial substrate, the stamping duration, and the concentration of the patterned molecules. Microcontact insertion printing enables dilute and isolated molecules to be patterned within a background SAM matrix, in a way that is not possible by other lithographic strategies (Mullen et al. 2007a; Srinivasan et al. 2007). Diffusion of the inserted molecules is also prevented, as in $\mu \mathrm{DP}$. Figure $4 \mathrm{~b}$ depicts the $\mu \mathrm{CIP}$ process. Initially, a patterned molecularly coated elastomeric stamp is brought into contact with a substrate coated with a preexisting monolayer that is not easily displaced; the molecules on the stamp then insert into the defects in the monolayer only in places where the stamp and substrate are in contact (Bumm et al. 1996; Cygan et al. 1998). Together, these soft-lithographic strategies offer straightforward, versatile, and low-cost methods to fabricate chemical patterns over large areas without the requirement of specialized facilities.

Both LACP and soft lithography can be employed to fabricate functionalized surfaces that exhibit selective molecular recognition. In most cases, fabrication of these surfaces begins with a SAM deposited across a $\mathrm{Au}$ substrate engineered to resist non-specific protein adsorption (Prime and Whitesides 1993; Wang et al. 1997; Sigal et al. 1998; Ostuni et al. 2001). In some applications, where proteins or peptides are eventually anchored to SAMs, low percentages of tether molecules are codeposited to form a mixed monolayer. Large biomolecules are then either covalently bound to tethers or specifically oriented by high-affinity linkers (Lahiri et al. 1999a; Lahiri et al. 1999b; Yousaf and Mrksich 1999; Hodneland et al. 2002). In other applications, precursor tether molecules with distinct 
terminal functionalities from the preexisitng SAM are inserted into its defect sites (Weck et al. 1999; Mullen et al. 2007b; Shuster et al. 2008). Subsequently, the terminal groups of the tether molecules are functionalized with small-molecule ligands. This type of biospecific surface is currently being developed to separate proteins from biological mixtures (e.g., tissue homogenates, serum, etc.), to elucidate molecular interactions between known proteins and their smallmolecule targets, and to identify unknown proteins that bind selectively to small-molecule ligands (Mullen et al. 2007b; Mrksich 2008; Shuster et al. 2008). Further, these surfaces are not limited to functionalization with small biomolecules, but can present other small molecules such as chemical warfare agents and toxic industrial chemicals. In addition to patterning biologically relevant molecules, surfaces bearing tethers with different classes of functional groups can be created, enabling the attachment of many different small molecules, particles, and clusters.

In our recent work, we have utilized this scheme to fabricate chemical films of the neurotransmitter serotonin for creating small-molecule functionalized surfaces. These surfaces have been employed to demonstrate biospecific recognition by serotonin-specific antibodies (Shuster et al. 2008). Figure 5a and b shows inserted tether molecules isolated within a oligo(ethylene glycol)-terminated SAM before and after functionalization of the tether carboxylic acid terminal group with serotonin via EDC/NHS coupling chemistry (Hermanson 1996). Not only were these surfaces shown to discriminate between the capture of proteins specific for serotonin versus those specific for other closely related small molecules, such as dopamine, but they also prevented non-specific recognition of "ubiquitous" proteins such as fibrogen, fibronectin, and bovine serum albumin. The ability to discriminate between large biomolecule binding partners based on their specific non-covalent interactions sets the stage for the creation of multiplexed surfaces, as shown schematically in Figure 5c, where a number of isolated small-molecule probes can be sequentially patterned on the same surface in order to capture multiple types of biomolecules based on their interactions in a competitive environment. It is important to note that in the overlapping patterned regions, the tether molecules (which are functionalized with smallmolecule probes) are isolated such that they do not interact with other probe molecules in the surrounding region. This molecular isolation can be controlled and influenced by many factors and has been confirmed by both ensemble and localized analytical techniques (Mullen et al. 2007a; Shuster et al. 2008).

Current traditional lithographic techniques are not capable of producing chemical patterns with this level of precision. Serial techniques have been devised to manipulate and to arrange single atoms as well as to draw molecular patterns on a surface with a scanning probe tip (Eigler and Schweizer 1990; Weiss and Eigler 1992, 1993; Piner et al. 1999; Xu et al. 1999). Although recent work has made creative and inventive efforts to create massive arrays of tips, these techniques require further development and will likely be utilized for specialized applications in analogy to electronbeam lithography (Salaita et al. 2006; Mirkin 2007). A key advantage of LACP, $\mu$ CIP, and related techniques is the ability to fabricate chemical films of isolated single molecules or bundles of molecules diluted within a background matrix over large areas. The molecular composition of the chemical pattern is influenced by the extent of insertion of the patterned molecules, which can be controlled by tuning the patterning duration, the concentration of the patterned molecules, the quality of the preexisting SAM, and the intermolecular interaction strengths of both the preexisting SAM and the patterned or inserted molecules (Mullen et al. 2007a). This control of molecular composition and isolation is particularly critical for small-molecule functionalized surfaces. If tether molecules linked to small-molecule probes are spaced too closely, non-specific binding increases; whereas if derivatized tethers are spaced too far apart, then the efficiency of capturing large biomolecules is diminished.

It is also important to anticipate the limitations of these hybrid approaches for fabricating patterned small molecules. First, the small-molecule probe must be covalently coupled to the patterned tether molecule via a functional group. This covalent attachment could hinder recognition and capture of larger target analytes. Another limitation of this hybrid strategy is that the patterned molecules are statistically distributed rather than directly placed. This could result in under-utilized areas where no patterned molecules are present or regions where clusters of molecules are inserted. Despite such limitations, insertion self-assembly and $\mu$ CIP have the potential to be applied to a wide variety of chemical systems and to enable capabilities not previously possible. 
(a)

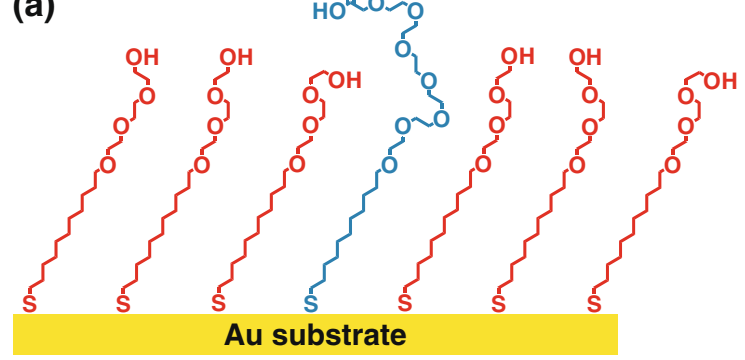

(b)
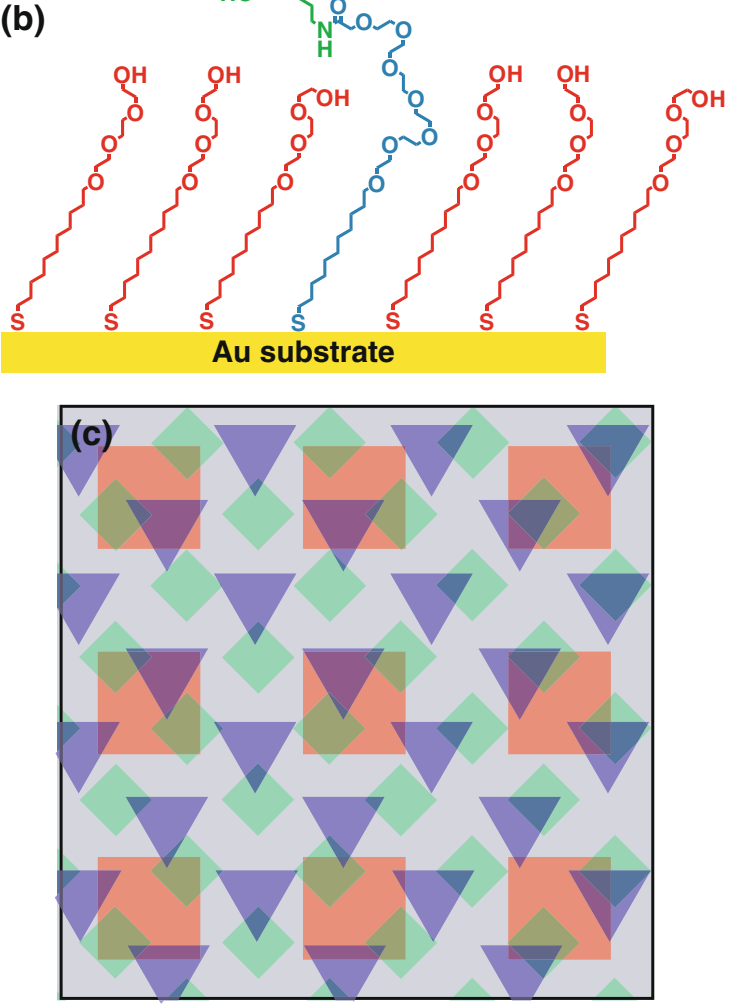

Fig. 5 Patterning Diluted Biospecific Small-Molecule Probe Surfaces. Inserted tether molecules isolated within an oligo(ethylene glycol)-terminated self-assembled monolayer (a) before and (b) after selective functionalization of carboxylic acid terminal group with serotonin via $\mathrm{N}$-(3-dimethylaminopropyl)N'-ethylcarbodiimide (EDC)/N-hydroxysuccinimide (NHS) coupling chemistry. (c) Schematic of a multiplexed surface created by multiple patterning steps where each color/shape represents a different small-molecule ligand

\section{Future prospects and conclusions}

When Moore first noted the scaling of semiconductor devices 40 years ago, he could not have imagined the influence and implications it would have on applications outside the semiconductor industry. Incremental improvements continue in the manufacturing processes used in the mature and established semiconductor industry. However, with the hybrid techniques highlighted in this perspective, along with similar strategies, it will become routine to fabricate, to manipulate, and to visualize structures at the 1-50 nm supramolecular scale. For example, hybrid patterning techniques enable access to biological systems with unprecedented molecular precision, allowing for the study and characterization of single and groups of biomolecules in various environments. With this structural and functional resolution of biomolecules, the underlying mechanisms can begin to be understood, enabling structures and devices to be developed and fabricated on the same scales as biological systems. These systems can then be mimicked, engineered, and improved.

The underlying theme for this perspective has been that there is no universal strategy or technique for every application; rather, for each application, there are several possible methods to create patterned surfaces, most of which are just now being developed. It is important to understand the specific requirements and how they relate and can be applied to each technique's advantages and limitations. It is likewise critical to develop the metrology tools and methods to test and to optimize the patterns created. All these hybrid techniques stem from the idea that molecular assembly can be coupled to the fidelity and sophistication of lithography. As our understanding and control of self- and directed assembly increase, the stringency of the underlying lithography will diminish, enabling wider access to and larger utilization for a greater number of applications.

Acknowledgments We thank Prof. Jennifer Hampton, Dr. Arrelaine Dameron, and Dr. Mary Elizabeth Anderson for insightful discussions. TJM is grateful for a fellowship from the American Chemical Society Division of Analytical Chemistry sponsored by the Society for Analytical Chemists of Pittsburgh. The research of the authors is supported by the Penn State Materials Research Institute, Penn State Nanofabrication Facility, and the Penn State Center for Nanoscale Science, an NSF-funded Materials Research Science and Engineering Center. Parts of this work were conducted at the Penn State node of the NSF-funded National Nanotechnology Infrastructure Network.

\section{References}

Allara DL, Nuzzo RG (1985) Spontaneously organized molecular assemblies. 2. Quantitative infrared spectroscopic 
determination of equilibrium structures of solution-adsorbed normal-alkanoic acids on an oxidized aluminum surface. Langmuir 1:52-66

Anderson ME, Srinivasan C, Hohman JN et al (2006) Combining conventional lithography with molecular self-assembly for chemical patterning. Adv Mater 18:3258-3260

Bent SF (2007) Heads or tails: which is more important in molecular self-assembly? ACS Nano 1:10-12

Black CT (2007) Polymer self-assembly as a novel extension to optical lithography. ACS Nano 1:147-150

Brunner TA (2003) Why optical lithography will live forever. J Vac Sci Technol B 21:2632-2637

Bumm LA, Arnold JJ, Cygan MT et al (1996) Are single molecular wires conducting? Science 271:1705-1707

Bumm LA, Arnold JJ, Charles LF et al (1999) Directed selfassembly to create molecular terraces with molecularly sharp boundaries in organic monolayers. J Am Chem Soc 121:8017-8021

Chen CS, Mrksich M, Huang S et al (1997) Geometric control of cell life and death. Science 276:1425-1428

Cygan MT, Dunbar TD, Arnold JJ et al (1998) Insertion, conductivity, and structures of conjugated organic oligomers in self-assembled alkanethiol monolayers on $\mathrm{Au}\{111\}$. J Am Chem Soc 120:2721-2732

Dameron AA, Charles LF, Weiss PS (2005a) Structures and displacement of 1-adamantanethiol self-assembled monolayers on $\mathrm{Au}\{111\}$. J Am Chem Soc 127:8697-8704

Dameron AA, Hampton JR, Smith RK et al (2005b) Microdisplacement printing. Nano Lett 5:1834-1837

Dameron AA, Hampton JR, Gillmor SD et al (2005c) Enhanced molecular patterning via microdisplacement printing. J Vac Sci Technol B 23:2929-2932

Daniel TA, Uppili S, McCarty G et al (2007) Effects of molecular structure and interfacial ligation on the precision of cu-bound alpha, omega-mercaptoalkanoic acid "molecular ruler" stacks. Langmuir 23:638-648

Delamarche E, Schmid H, Bietsch A et al (1998) Transport mechanisms of alkanethiols during microcontact printing on gold. J Phys Chem B 102:3324-3334

Eigler DM, Schweizer EK (1990) Positioning single atoms with a scanning tunneling microscope. Nature 344:524-526

Evans SD, Ulman A, Goppertberaraducci KE et al (1991) Selfassembled multilayers of omega-mercaptoalkanoic acids selective ionic interactions. J Am Chem Soc 133:5866-5868

Gates BD, Xu QB, Stewart M et al (2005) New approaches to nanofabrication: molding, printing, and other techniques. Chem Rev 105:1171-1196

Haes AJ, Zou S, Schatz GC et al (2004) A nanoscale optical biosensor: the long range distance dependence of the localized surface plasmon resonance of noble metal nanoparticles. J Phys Chem B 108:109-116

Hammond PT (2004) Form and function in multilayer assembly: new applications at the nanoscale. Adv Mater 16:1271-1293

Hatzor A, Weiss PS (2001) Molecular rulers for scaling down nanostructures. Science 291:1019-1020

Hatzor de Picciotto A, Wisser-Gross AD, Lavallee G et al (2007) Arrays of $\mathrm{Cu}^{2+}$-complexed organic clusters grown on gold nano dots. J Exp Nanosci 2:3

Hawker CJ, Russell TP (2005) Block copolymer lithography: merging "bottom-up" with "top-down" processes. MRS Bull 30:952-966
Henzie J, Barton JE, Stender CL et al (2006) Large-area nanoscale patterning: chemistry meets fabrication. Acc Chem Res 39:249-257

Hermanson GT (1996) Bioconjugate techniques. Academic Press, San Diego

Hodneland CD, Lee YS, Min DH et al (2002) Selective immobilization of proteins to self-assembled monolayers presenting active site-directed capture ligands. Proc Natl Acad Sci USA 99:5048-5052

Jager B, Schurmann H, Muller HU et al (1997) X-ray and low energy electron induced damage in alkanethiolate monolayers on Au-substrates. Z Phys Chem 202:263-272

Kumar A, Whitesides GM (1993) Features of gold having micrometer to centimeter dimensions can be formed through a combination of stamping with an elastomeric stamp and an alkanethiol ink followed by chemical etching. Appl Phys Lett 63:2002-2004

Kumar A, Biebuyck HA, Whitesides GM (1994) Patterning self-assembled monolayers - applications in materials science. Langmuir 10:1498-1511

Lahiri J, Isaacs L, Tien J et al (1999a) A strategy for the generation of surfaces presenting ligands for studies of binding based on an active ester as a common reactive intermediate: a surface plasmon resonance study. Anal Chem 71:777-790

Lahiri J, Ostuni E, Whitesides GM (1999b) Patterning ligands on reactive sams by microcontact printing. Langmuir 15:2055-2060

Lewis PA, Donhauser ZJ, Mantooth BA et al (2001a) Control and placement of molecules via self-assembly. Nanotechnology 12:231-237

Lewis PA, Smith RK, Kelly KF et al (2001b) The role of buried hydrogen bonds in self-assembled mixed composition thiols on Au\{111\}. J Phys Chem B 105:10630-10636

Lopez GP, Biebuyck HA, Frisbie CD et al (1993) Imaging of features on surfaces by condensation figures. Science 260:647-649

Love JC, Estroff LA, Kriebel JK et al (2005) Self-assembled monolayers of thiolates on metals as a form of nanotechnology. Chem Rev 105:1103-1169

Lundstrom M (2003) Moore's law forever? Science 299: 210-211

Mannering VH, Hodge A (2007) Gross domestic product and corporate profits. Department of Commerce, pp 1-15

Mansky P, Liu Y, Huang E et al (1997) Controlling polymersurface interactions with random copolymer brushes. Science 275:1458-1460

Mirkin CA (2007) The power of the pen: development of massively parallel dip-pen nanolithography. ACS Nano 1:79-83

Moore GE (1965) Cramming more components onto integrated circuits. Electronics 38:114-117

Moore GE (1995) Lithography and the future of Moore's law. Proc SPIE 2447:2-17

Mrksich M (2008) Mass spectrometry of self-assembled monolayers: a new tool for molecular surface science. ACS Nano 2:7-18

Mullen TJ, Dameron AA, Weiss PS (2006) Directed assembly and separation of self-assembled monolayers via electrochemical processing. J Phys Chem B 110:14410-14417

Mullen TJ, Srinivasan C, Hohman JN et al (2007a) Microcontact insertion printing. Appl Phys Lett 90:063114 
Mullen TJ, Dameron AA, Andrews AM et al (2007b) Selecting and driving monolayer structures through tailored intermolecular interactions. Aldrichimica Acta 40:19-31

Nuzzo RG, Dubois LH, Allara DL (1990) Fundamental-studies of microscopic wetting on organic-surfaces. 1. Formations and structural characterization of a self-consistent series of polyfunctional organic monolayers. J Am Chem Soc 112:558-569

Ostuni E, Chapman RG, Holmlin RE et al (2001) A survey of structure-property relationships of surfaces that resist the adsorption of protein. Langmuir 17:5605-5620

Park M, Harrison C, Chaikin PM et al (1997) Block copolymer lithography: periodic arrays of similar to $10^{11}$ holes in 1 square centimeter. Science 276:1401-1404

Pertsin AJ, Grunze M (1994) Low-energy structures of a monolayer of octadecanethiol self-assembled on $\mathrm{Au}(111)$. Langmuir 10:3668-3674

Piner RD, Zhu J, Xu F et al (1999) "Dip-pen" nanolithography. Science 283:661-663

Poirier GE, Pylant ED (1996) The self-assembly mechanism of alkanethiols on $\mathrm{Au}(111)$. Science 272:1145-1148

Porter MD, Bright TB, Allara DL et al (1987) Spontaneously organized molecular assemblies. 4. Structural characterization of normal-alkyl thiol monolayers on gold by optical ellipsometry, infrared-spectroscopy, and electrochemistry. J Am Chem Soc 109:3559-3568

Prime KL, Whitesides GM (1993) Adsorption of proteins onto surfaces containing end-attached oligo(ethylene oxide) - a model system using self-assembled monolayers. J Am Chem Soc 115:10714-10721

Rai-Choudhury P (1997) Handbook of microlithography, micromachining, and microfabrication. SPIE Optical Engineering Press, London

Rogers JA, Nuzzo RG (2005) Recent progress in soft lithography. Mater Today 8:50-56

Saavedra HM, Mullen TJ, Zhang PP et al (2008) Hybrid strategies in nanolithography. Rep Prog Phys (in preparation)

Salaita K, Amarnath A, Maspoch D et al (2005) Spontaneous "phase separation" of patterned binary alkanethiol mixtures. J Am Chem Soc 127:11283-11287

Salaita K, Wang YH, Fragala J et al (2006) Massively parallel dip-pen nanolithography with 55000-pen two-dimensional arrays. Angew Chem Intl Ed 45:7220-7223

Shuster MJ, Vaish A, Szapacs ME et al (2008) Biospecific recognition of tethered small molecules diluted in selfassembled monolayers. Adv Mater 20:164-167

Sigal GB, Mrksich M, Whitesides GM (1998) Effect of surface wettability on the adsorption of proteins and detergents. J Am Chem Soc 120:3464-3473

Smith RK, Reed SM, Lewis PA et al (2001) Phase separation within a binary self-assembled monolayer on $\mathrm{Au}\{111\}$ driven by an amide-containing alkanethiol. J Phys Chem B 105:1119-1122

Smith RK, Lewis PA, Weiss PS (2004) Patterned self-assembled monolayers. Prog Surf Sci 75:1-68

Srinivasan C, Mullen TJ, Hohman JN et al (2007) Scanning electron microscopy of nanoscale chemical patterns. ACS Nano 1:191-201
Stoykovich MP, Muller M, Kim SO et al (2005) Directed assembly of block copolymer blends into nonregular device-oriented structures. Science 308:1442-1446

Stoykovich MP, Nealey PF (2006) Block copolymers and conventional lithography. Mater Today 9:20-29

Stoykovich MP, Kang H, Daoulas KC et al (2007) Directed self-assembly of block copolymers for nanolithography: fabrication of isolated features and essential integrated circuit geometries. ACS Nano 1:168-175

Stranick SJ, Parikh AN, Tao YT et al (1994) Phase-separation of mixed-composition self-assembled monolayers into nanometer-scale molecular domains. J Phys Chem 98:7636-7646

Tanaka Y, Taguchi T, Fujii K et al (1998) $130 \mathrm{~nm}$ and $150 \mathrm{~nm}$ line-and-space critical-dimension control evaluation using XS-1 x-ray stepper. J Vac Sci Technol B 16:3509-3514

Tanaka H, Anderson ME, Horn MW et al (2004) Positionselected molecular ruler. Jpn J Appl Phys Part 2 Lett Express Lett 43:L950-L953

Thompson SE, Parthasarathy S (2006) Moore's law: the future of Si microelectronics. Mater Today 9:20-25

Thurn-Albrecht T, Steiner R, DeRouchey J et al (2000) Nanoscopic templates from oriented block copolymer films. Adv Mater 12:787-791

Tiberio RC, Craighead HG, Lercel M et al (1993) Selfassembled monolayer electron-beam resist on GaAs. Appl Phys Lett 62:476-478

Wang RLC, Kreuzer HJ, Grunze M (1997) Molecular conformation and solvation of oligo(ethylene glycol)-terminated self-assembled monolayers and their resistance to protein adsorption. J Phys Chem B 101:9767-9773

Weck M, Jackiw JJ, Rossi RR et al (1999) Ring-opening metathesis polymerization from surfaces. J Am Chem Soc 121:4088-4089

Weiss PS, Eigler DM (1992) Adsorption and accommodation of Xe on Pt $\{111\}$. Phys Rev Lett 69:2240-2243

Weiss PS, Eigler DM (1993) What is underneath? Moving atoms and molecules to find out. In: Nanosources and manipulations of atoms under high fields and temperatures: applications, NATO ASI Series E: Appl Sci 235: 213-217, Kluwer Academic, Dordrecht

Xia Y, Whitesides GM (1998a) Soft lithography. Annu Rev Mater Sci 28:153-184

Xia YN, Whitesides GM (1998b) Soft lithography. Angew Chem Intl Ed 37:551-575

Xia YN, Rogers JA, Paul KE et al (1999) Unconventional methods for fabricating and patterning nanostructures. Chem Rev 99:1823-1848

Xu S, Miller S, Laibinis PE et al (1999) Fabrication of nanometer scale patterns within self-assembled monolayers by nanografting. Langmuir 15:7244-7251

Yousaf MN, Mrksich M (1999) Diels-Alder reaction for the selective immobilization of protein to electroactive selfassembled monolayers. J Am Chem Soc 121:4286-4287 